\title{
LOS INGENIEROS MILITARES Y LA ADECUACIÓN DE CÁDIZ PARA LA VISITA DE ISABEL II
}

\author{
PEDRO CRUZ FREIRE | UNIVERSIDAD DE CÁDIZ
}

ORCID iD: 0000-0003-3965-4717

\begin{abstract}
RESUMEN
El presente artículo pretende analizar las medidas adoptadas por la Dirección y Comandancia de los Ingenieros militares andaluces para adecuar la ciudad de Cádiz a la visita de Isabel II. En este sentido, se analiza las diferentes actuaciones llevadas a cabo en los principales edificios de carácter militar de la capital gaditana, cuyo conjunto no solo se encontraba en un estado mejorable, sino que fue necesario actuar, pues conforma una de las grandes señas de identidad del patrimonio histórico de la ciudad.
\end{abstract}

PALABRAS CLAVE

Ingeniería Militar, fortificación, Isabel II, Castillo de San Sebastián, Cádiz.

\section{MILITARY ENGINEERING AND CADIZ'S ADEQUACY FOR ISABEL II VISIT}

\begin{abstract}
This article aims to analyze the measures adopted by the Corp of Andalusian Military Engineers to adapt the city of Cádiz to Isabel II's visit. In this sense, different actions carried out in the main military buildings of the city are analyzed. Those works showed that these buildings needed a deep restoration and better care, given its importance as an identity of the historical heritage of the city.
\end{abstract}

\section{KEYWORDS}

Military engineering, fortifications, Isabel II, San Sebastian Castle, Cádiz. 


\section{CÁDIZ EN EL SIGLO XIX Y LA VISITA DE LA REINA ISABEL II}

$\mathrm{E}$

n un intento por adecuar a la institución monárquica a una situación social cambiante, donde ahora primaba un pensamiento liberal de la sociedad, la monarquía buscó nuevas fórmulas que estrecharan las relaciones con su pueblo. La pérdida de súbditos españoles hacia la Corona desembocó en la realización de un viaje por diferentes ciudades y localizaciones que persiguió la consolidación de la figura real y el acercamiento de la institución a los diferentes estratos sociales ${ }^{1}$.

En septiembre de 1862, la reina Isabel comenzó un intenso viaje en el que recorrió algunas de las localizaciones más significativas de Andalucía y Murcia. El viaje comenzaría el 12 de septiembre y se alargó hasta la última fecha de octubre, tiempo en el que visitó las provincias de Jaén, Córdoba, Sevilla, Cádiz, Granada, Málaga, Almería y Murcia. Con ella viajaron sus dos hijos mayores, el Duque de Tetuán, presidente del consejo de ministros, el ministro de estado Saturnino Calderón y el ministro de Fomento marqués de la Vega. Además, la acompañaron todo un séquito de mayordomos, caballerizos, médicos y personas de su estrecha confianza.

La expectación generada por la visita real provocó toda una serie de propuestas para embellecer la ciudad. Desde luego, Cádiz debía engalanarse para una celebración a todas luces atípica en la capital andaluza, por lo que se presentaba como una oportunidad extraordinaria para volver a brillar bajo los ojos del panorama nacional. Para ello, la ciudad se atavió con sus mejores galas y, como ha mostrado el fotógrafo galés Charles Clifford, fueron numerosas las arquitecturas efímeras erigidas en los puntos más destacados de la ciudad ${ }^{2}$. En este sentido, uno de los principales focos a ser explotados como seña de identidad y reflejo de la sociedad gaditana debía ser su idiosincrasia militar, encarnada en los diferentes ejemplos de arquitectura fortificada, única en el contexto español y ejemplo de primer nivel en el ámbito europeo e iberoamericano. Las diferentes instituciones militares, tanto locales como andaluzas, sumaron esfuerzos para dotar a la ciudad de mejoras en sus infraestructuras para tal fin. Fruto de ello se ha conservado un rastro de documentación que ha permitido identificar las principales cuestiones abordadas por el colectivo militar, las deficiencias de un patrimonio histórico de sumo valor para la ciudad y las obras más relevantes llevadas a cabo.

1 NÚÑEZ GARCÍA, Víctor Manuel. Monarquía y nación a través de la visita de Isabel II a Andalucía en 1862. La dimensión cultural de las ceremonias reales, Hispania: Revista española de historia, 79, 2019. Pp. 331-357

2 Las fotografías tomadas por Charles Clifford pueden consultarse en los siguientes trabajos: FONTANELLA, Lee. Clifford en España: un fotógrafo en la corte de Isabel II. Madrid, El Viso, 1999. GARÓFANO SÁNCHEZ, Rafael. Cádiz en la fotografía del siglo XIX. Cádiz: Unicaja, Obra Socio Cultural, D.L. 1993. 


\section{LA FINANCIACIÓN DE LOS TRABAJOS}

Si bien se tenía claro cuáles eran los puestos que necesitaban ser reparados y decorados para la visita, había más dudas sobre la manera de financiarlos. En carta de Miguel de Santillana, Coronel Comandante de Ingenieros de Cádiz, fechada a 3 de septiembre, el militar aconsejaba a los diferentes cuerpos militares que se

"verifique los gastos puramente indispensables para que tanto en la capital como en la Plaza de Cádiz se adornen los edificios ocupados por el cuerpo de tal modo que obteniéndose todo el decoro $\gamma$ lucimiento necesario $\gamma$ que este en armonía con lo que ejecuten las demás corporaciones, se procure sin embargo la mayor economía posible ${ }^{\prime \prime}$.

Las dudas sobre la procedencia de los fondos aplicados a dichas reparaciones fueron consultadas al ministerio de Guerra. El ministro informaba al Capitán General de Andalucía que los gastos que fuesen ocasionados por su viaje no debían de ninguna manera obtenerse de los diferentes salarios de los oficiales y la tropa, pues Su Majestad no deseaba que los individuos del ejército sufran el menor gravamen" ${ }^{4}$. Tras una variada correspondencia entre los diferentes cuerpos andaluces y el ministerio se decidió lo siguiente. En primer lugar, los reparos que no fuesen ocasionados exclusivamente por la visita de la Reina, relativos al blanqueo de paredes y obras de mantenimiento, se financiasen con las partidas mensuales que ya tenían asignadas dichas instituciones para tal fin, lo cual resultó en un gasto de 17.040 reales, aprobado por Isabel II en carta de 24 de enero de $1863^{5}$. Por su parte, los gastos aprobados expresamente para el evento serían sufragados por partidas extraordinarias abonadas por la Corona.

\section{LA LABOR DE LOS INGENIEROS MILITARES DURANTE LA VISITA}

Las crónicas de la época relativas al viaje de Isabel II, especialmente las ejecutadas por Pongilioni e Hidalgo y Cos-Gayón, reflejan con acierto las intervenciones realizadas por los ingenieros militares. En ese sentido, son interesantes las apreciaciones de ambos autores

\footnotetext{
3 Archivo Histórico Provincial de Cádiz (De aquí en adelante, AHPC). Fondo Pettengui, sig. 35.657/13, s/f.

4 Ídem.

5 Real Orden de 6 de enero de 1863.
} 
sobre la llegada de la Reina a la capital gaditana. En primer lugar, Pongilioni e Hidalgo expusieron al respecto:

"La reina constitucional de España, al contemplar desde el anchuroso océano a Cádiz, debió recordar sin duda que se acercaba al que fue baluarte de la independencia española en la época más gloriosa de nuestra historia moderna [...] $\gamma$ al verla desde lejos tan bella $\gamma$ engalanada para recibirla dignamente, debió también exclamar, como lo hicieron algunos de sus augustos predecesores, Esa es Cádiz, mi ciudad querida"'b.

Por su parte, Cos-Gayón afirmaba, en formas similares, lo siguiente:

“En aquel día Cádiz, la histórica, la rica, la hermosa, la fuerte, aparecía más encantadora que nunca, al presentarse al viajero sobre las aguas tranquilas del océano, bañada en los resplandores del sol no velados por nube alguna"7.

Desde agosto de 1862 se estuvo trabajando sin descanso en los preparativos para la celebración. En carta de 21 de agosto se despachó una orden para preparar la ciudad por parte del capitán General de Cádiz. En este sentido, el capitán aconsejaba la terminación de las prendas de vestuario acordes a la celebración, así como el equipo y la montura necesarias para los caballos que desfilarían en diversos actos. En segundo lugar, sugería a los diferentes directores que ejecutasen una relación de gastos que fuesen necesarios para la limpieza, adorno de fachadas e iluminación de los diferentes cuarteles de la ciudad.

El 18 de agosto, desde la dirección de la Subinspección de ingenieros, se denunciaba la escasa atención volcada, no solo sobre los cuarteles destinados a los ingenieros militares, sino también a las propias fortificaciones. Desde esta misiva se instaba además a arreglary preparar convenientemente caminos, escaleras provisionales y demás obras de acceso que permitiesen a los visitantes recorrer las diferentes obras y edificios.

6 PONGILIONI, Arístides HIDALGO, Francisco de P.M. Crónica del viage de SS. MM. $\gamma$ AA. RR. á las provincias de Andalucía en 1862. Cádiz, Eduardo Cautier (impresor), 1863, p. 173.

7 COS-GAYÓN, Fernando. Crónica del viaje de sus Majestades y Altezas Reales á Andalucía y Murcia en septiembre y octubre de 1862 (1863). Madrid, Imprenta Nacional, 1863, p. 141. 
Conjuntamente a ello, otra misiva indicaba la solicitud de cien caballos para prestar servicio durante la estancia de la comitiva real, lo que obligaría por su parte a crear una serie de pesebres provisionales en diferentes cuarteles de la ciudad para el correcto cuidado de los equinos.

Una vez resuelto la manera de sufragar los gastos, se llevó a cabo un informe relativo a los edificios y localizaciones que debían estar sujetos a mejoras y actualizaciones, los cuales fueron detallados un documento titulado Cuenta de los gastos originados en los festejos hechos en la visita hecha a esta plaza por SSMM ${ }^{8}$. Un informe remitido por el coronel de ingenieros de la ciudad de Cádiz días después de la finalización de la visita de la Reina en la capital. En el documento se especifican los trabajos que fueron necesarios para acomodar los emplazamientos susceptibles de ser visitados por la comitiva real y que complementarían las actividades programadas desde la corona en la plaza. En este sentido, se detallan los trabajos efectuados en el castillo de San Sebastián, en varios cuarteles y almacenes de pólvora de la ciudad y en otras localizaciones militares de la vecina localidad de San Fernando, los cuales serán analizados a continuación.

Situado en un islote de uno de los extremos de la playa de la Caleta, el Castillo de San Sebastián es uno de los principales guardianes defensivos de la capital gaditana. Erigido en un lugar en donde existía una ermita dedicada a San Sebastián y, bastante tiempo atrás, un templo dedicado al dios Cronos, el edificio que vemos en la actualidad data de una reforma comenzada a principios del siglo XVIII ${ }^{9}$. En planta, forma un eneágono irregular con dos ángulos entrantes. Por su parte, su perímetro estaba provisto de parapeto con cañoneras y foso, comunicando su interior con dos puentes levadizos. En su interior, una plaza de armas con las dependencias habituales de este tipo de estructuras defensivas, para el descanso de la tropa y el almacenaje de los pertrechos. Toda la isla estaba protegida por un parapeto en todo su perímetro, excepto en el frente sur, que contaba con una considerable defensa natural. Desde finales del siglo XVIII, aunque con modificaciones posteriores, se conectó con tierra gracias al canal de comunicación que se conserva en la actualidad, cuya fábrica pertenece al ingeniero Juan Caballero ${ }^{10}$.

8 Cuenta de los gastosoriginados en losfestejoshechos en la visita hecha a esta plaza porSSMM. AHPC. Fondo Pettengui, sig, 35.657/13,s/f. 9 Para una información más precisa sobre esta construcción, pueden consultarse los siguientes trabajos. FERNÁNDEZ CANO, Víctor. Las defensas de Cádiz durante la Edad Moderna. Sevilla: Escuela de Estudios Hispano-Americanos, 1974. CALDERÓN QUIJANO,José Antonio:Cartografía military marítima de Cádiz: 1513-1878. Sevilla: Escuela de Estudios Hispano-Americanos, 1978.

10 El mejor documento que nos ha llegado hasta hoy sobre cómo se encontraba el castillo en el momento de la visita de la reina Isabel II lo debemos al ingeniero Juan Caballero: “En el mes de octubre de 1770 de orden de Su Magestad comunicada al excelentísimo Señor Marques de Vanmarcke se empezó este Camino provicional para socorro del Castillo de S[a]n Sebastian" Archivo General de Simancas. Mapas, planos y dibujos, sig. 11, 139. Puede verse reproducido en MUÑOZ CORBALÁN, Juan Miguel: La imagen versátil de la ciudad fortificada: cartografía fantaseada hispánica en los siglos XVIXVIII. Revista de Història da Arte, 13, 2018, p. 174. También puede consultarse en CHÍAS, Pilar, ABAD, Tomás (Dirs.). El patrimonio fortificado. Cádiz y el Caribe: una relación transatlántica. Alcalá de Henares: Universidad de Alcalá, 2011, p. 28. 
Desde la Subinspección de Ingenieros se entendió que habría pocos enclaves más acertados que el castillo de San Sebastián para representar la magnitud de la historia militar gaditana. Además de su condición de prototipo destacado de arquitectura militar, su privilegiada situación geográfica ofrecía una vista inmejorable del conjunto de la ciudad. Razones de peso para que desde un primer momento se valorase la idea de adecuar convenientemente la fortaleza para la visita real. Los trabajos fueron organizados y coordinador por el ingeniero Juan Manuel Lombera, por aquel entonces Coronel de Ingenieros de la ciudad ${ }^{1}$.

En la relación de gastos para los festejos se justificaba cada una de las intervenciones llevadas a cabo en el castillo. En primer lugar, se acomodó el canal de acceso al castillo, asfaltándolo y decorándolo con banderas y gallardetes interpolados de escudos reales y trofeos. En el interior del conjunto, se dispuso una estructura de escaleras y galerías sobre las bóvedas del segundo piso, donde se construyó a la altura del parapeto un mirador, "para que SSMM vieran desde este punto toda la obra, así como las magníficas vistas a la mar y plaza de Cádiz". Además de otras obras menores de decoración en el fuerte, es interesante la creación de un álbum fotográfico de todo el conjunto del castillo, una serie de vistas que, unidas entre sí, presentaban una imagen completa de la fortaleza, de las que se hicieron varios ejemplares para la Corte y el Ministerio de Guerra. Fueron las obras en San Sebastián las más costosas para la administración militar gaditana, tasadas en 17.040 reales.

En la crónica de Cos-Gayón se especificaron detalladamente cada una de estas mejoras.

"Las más considerables construcciones que en este concepto se habían ejecutado en Cádiz, habían sido en el fuerte de san Sebastián, que penetrando bastante dentro del mar está unido a las murallas por un largo arrecife levantado sobre las aguas del Océano [...] Atravesaron SS.MM a pie el estrecho arrecife, a pesar de que estaban dispuestas sillas de mano, $\gamma$ aunque un fuerte viento hacia incómodo el tránsito. Vieron con detenimiento las grandes baterías bajas acasamatadas ya concluidas, $\gamma$ por escaleras provisiones llegaron a lo alto de la torre central en que había construido un balcón de madera para que pudiesen disfrutar del panorama extenso y grandioso que desde allí se descubre".

11 La más actualizada información sobre la labor de este ingeniero, la presenta el siguiente trabajo. HINAREJOS MARTÍN, Nuria, Aportaciones a la ingeniería militar del siglo XIX: la obra de Juan Manuel Lombera y Rivero (1818post. 1875). Actas de las segundas jornadas sobre historia, arquitectura $\gamma$ construcción fortificada. Madrid, 6-7 de octubre de 2016, Madrid, Instituto Juan de Herrera - Fundación Cárdenas - Centro de Estudios José Joaquín de Mora, 2016, págs. 325-342. 
Asimismo, también destacó el cronista la colección fotográfica realizada expresamente para la comitiva real:

"Las Personas Reales se sentaron delante de una mesa en que hallaban extendidos los planos de la fortaleza $\gamma$ vistas fotográficas de la misma, que examinaron" ${ }^{12}$.

\section{Ilustración 1}

Castillo de San Sebastián en el islote de la Caleta (Cádiz)

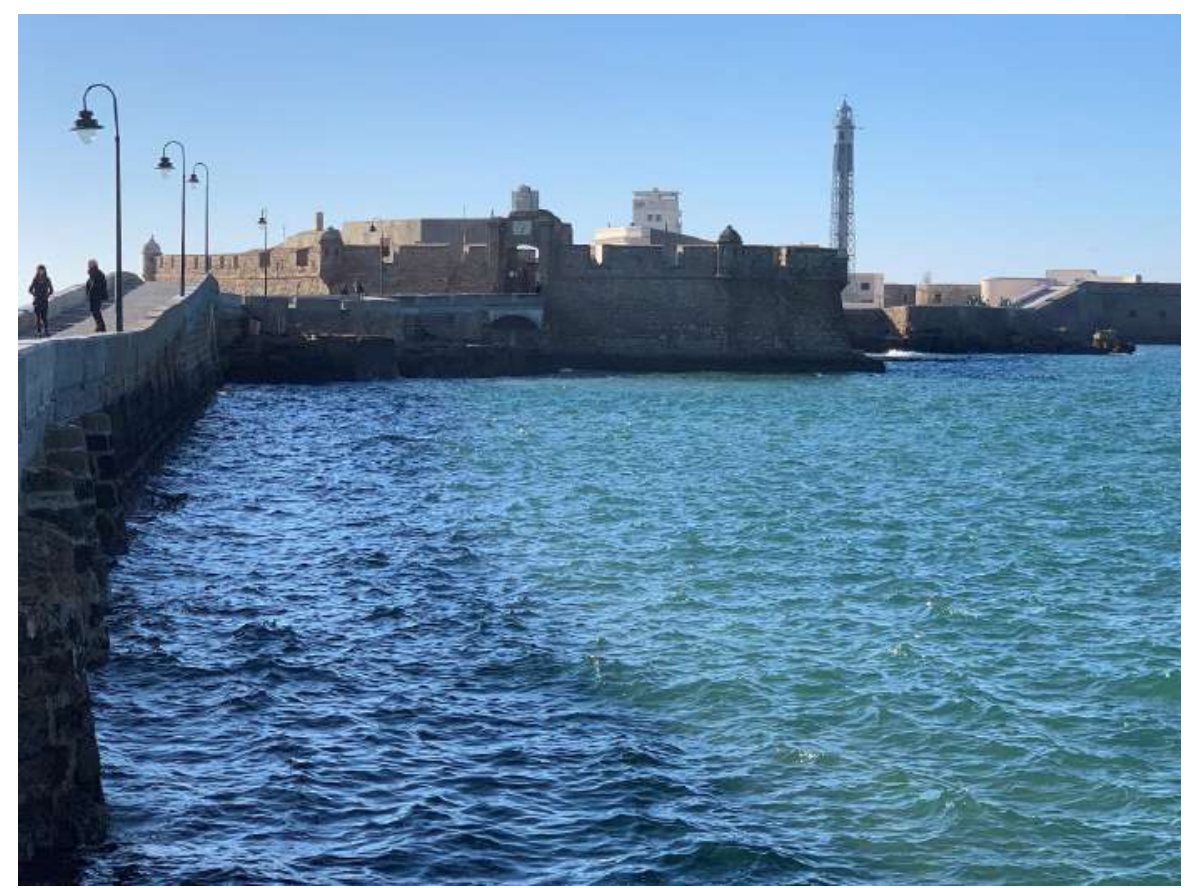

Igual de interesante resulta la visión que proporciona de este episodio los cronistas Pongilioni e Hidalgo. Si bien comienza haciendo un pequeño resumen de la historia y jerarquía del castillo en el sistema defensivo de la bahía gaditana, seguidamente continúa describiendo los pormenores de la recepción de la reina en la fortaleza. Previamente, el general o'Donnell había examinado con detenimiento las diferentes labores ejecutadas en el casti1lo, "de las que se mostró muy satisfecho", por lo que "rogó a S.M que visitase el castillo, cuya avanzada, en la cual se está construyendo la mayor de las nuevas obras acasamatadas, ha de llevar en adelante el nombre de Fortaleza Reina Isabel $\mathrm{II}^{\prime \prime}{ }^{13}$.

12 COS-GAYÓN: Crónica del viaje...Op. Cit. pp. 170-171.

13 PONGILIONI, HIDALGO: Crónica de su... Op.cit. p. 271. 
Como bien indica el cronista, la idea primitiva era que la comitiva real llegase al castillo por mar. Sin embargo, finalmente se optó por utilizar el camino de tierra. Se aprovechó la circunstancia de la baja pleamar para cruzar el canal que "acababa de ser reconstruido, tenía por esta circunstancia excelente piso y presentaba un cómodo camino" ${ }^{14}$. En el interior del fuerte visitaron las baterías bajas acasamatadas y desde allí continuaron al segundo piso donde, desde el balcón de madera provisional construido especialmente para la ocasión, se le presentaron las vistas a la ciudad y los diferentes planos y fotografías aludidas anteriormente. La recepción concluyó con otros actos de carácter oficial, en el que destaca la rebaja de pena de los presidiarios que trabajaron en la remodelación del castillo y la otorgación con la cruz de comendador de Carlos III al ingeniero Lombera por su excelente trabajo en el mismo.

\section{Ilustración 2}

\section{Panteón de Marinos Ilustres en la Población de San Carlos (San Fernando, Cádiz)}

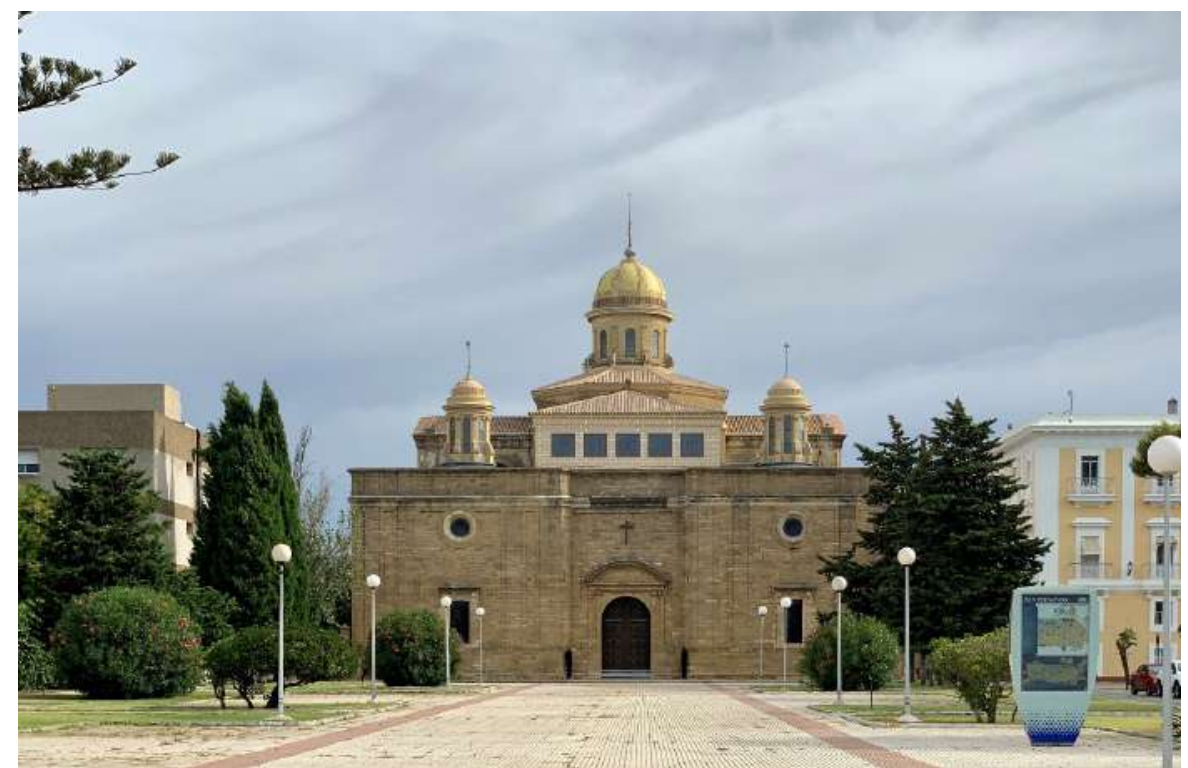

Como quedó dicho anteriormente, fue indispensable construir una serie de caballerizas para alojar a todo el conjunto de equinos que participarían en los diferentes desfiles en honor a la familia real. Para ello, se decidió que el lugar más apropiado sería la planta baja de los cuarteles de la Bomba. En este cuartel, ejecutado por el ingeniero Ignacio Sala en la década de 1730, se dispuso mediante orden de Juan de Quiroga, capitán de Ingenieros, que se sustituyeran las estancias bajas de la tropa por una serie de caballerizas que alojasen un total de cien caballos. Una obra provisional que el propio ingeniero excusaba en que sus 
materiales serían reutilizados en futuras obras del cuartel, dado su carácter provisional y sin ninguna aplicación futura para la plaza.

Los trabajos en la capital gaditana culminaron con una serie de obras menores realizadas en la fachada del baluarte de la Candelaria, con materiales procedentes del anexo pabellón de ingenieros situado frente a él, así como en los almacenes de pólvora de Jesús Nazareno y Nuestra Señora de Regla, donde presumiblemente la comitiva real se detendría a pasar revista a las tropas apostadas en la capital. Para ambos trabajos fueron destinados un total de 6.300 reales.

Una vez completada todas las visitas previstas en la ciudad de Cádiz, la reina y su comitiva zarparon desde la fortaleza de San Lorenzo del Puntal hacia la vecina localidad de San Fernando. Embarcados en el vapor Remolcador, especialmente adornado para la ocasión, se dirigieron en dirección al Arsenal de La Carraca mientras una serie de salvas de artillería despedía al cortejo, acompañado por varios buques de guerra. Los trabajos realizados dentro de las instalaciones militares de la ciudad se limitaron prácticamente al adorno de fachadas y adecuación de varios espacios públicos. Según informa Cos-Gayón, el muelle de la Carraca se había convertido en un elegante embarcadero:

"La gran plaza por donde se entra al arsenal, convertida en bello $\gamma$ vasto salón, concluía en dos columnas, engalanadas con banderas nacionales, coronas de laurel, trofeos de anclas, cañones $\gamma$ piezas dejarcias" 15 .

Tras ser recibida en La Carraca por las autoridades civiles y militares, la Comitiva Real se dirigió a la población militar de San Carlos. Este proyecto, ideado solo un siglo atrás por Francisco Sabatini, modificado por el ingeniero Vicente Imperial Digueri y finalmente proyectado por el marqués de Ureña, fue un proyecto urbanístico promocionado por Carlos III para reubicar de manera organizada el departamento de marina de aquella localidad. Esta población ilustrada contaba una serie de cuarteles para las guardias y batallones de marina, así como para la brigada de artillería y la escuela de pilotos, un teatro, una aduana, un hospital, una iglesia y una ensenada para pequeñas embarcaciones, entre otros edificios reservados para la administración ${ }^{16}$. A lo largo de esta parada se prestó especial atención en el hospital y la iglesia, para posteriormente continuar hacia el centro histórico de la ciudad, la calle real y el ayuntamiento.

15 COS-GAYÓN: Cronica del viaje... Op. Cit. p. 160.

16 Si se desea profundizar en este tema, debe consultarse el siguiente trabajo: TORREJÓN CHAVES, Juan. La nueva población de San Carlos en la Isla de León (1774-1806). Madrid: Ministerio de Defensa, 1988. 
Por la información recogida en los fondos del Archivo Provincial de Cádiz, uno de los puntos de interés de la visita a san Fernando sería el Puente Suazo, aunque por cuestiones de agenda finalmente no fuese visitado. Sin embargo, se sabe que se efectuaron algunos arreglos para este evento. En la cuenta de los gastos originados para los festejos figura una partida de 2682 reales invertidos, entre jornales y materiales, para el revocado, enlucido y blanqueado del camino del puente y su acceso, los distintos cuerpos de guardia y los muros de las empalizadas, los cuales también se habían pintado de color caoba ${ }^{17}$. Sin duda, la importancia de dicha estructura en el esqueleto defensivo de la bahía gaditana parecía pretexto suficiente para la visita, más si cabe después de su papel desempeñado en la Guerra de la Independencia. Lamentablemente, la apretada agenda real impidió su inspección.

Por último, al igual que no se produjo el reconocimiento del Puente Suazo, también quedó en deseo la exhibición prevista en la escuela de tiro en Torregorda. Situada a medio camino entre Cádiz y San Fernando, la torre y batería de Torregorda formaba parte del sistema de vigilancia costera desde las cuales podían transmitirse de manera eficaz advertencias de peligrosidad ante un ataque naval, fundamentalmente en conexión con la torre de Sancti Petri y el castillo de San Sebastián. Gracias a este sistema, el flanco atlántico quedaba completamente guarnecido si se avistaba cualquier partida enemiga en las proximidades marítimas. Como queda dicho, desde la Subinspección de ingenieros se destinó una modesta partida de 735 reales para repellar los parapetos y las cañoneras, "muy dañadas por la intemperie" 18 . Asimismo, se blanqueó la ya desaparecida torre y se arreglaron algunos desconchados.

\section{CONCLUSIONES}

Todo lo expuesto a lo largo de este trabajo ayuda a comprender la importancia que adquirió el espíritu militar de la ciudad de Cádiz como parte inherente de su historia e identidad. Las ligeras modificaciones que los diferentes cuerpos militares tuvieron que poner en marcha para un evento de tal repercusión nacional hablan del buen estado de conservación en el que se hallaban, exceptuando algunos retoques habituales para este tipo de eventos. Un celo sobre el patrimonio que hoy día debe perpetuarse mediante su cuidado, atención y puesta en valor como elemento singular de la historia, no solo gaditana, sino también española.

17. AHPC. Fondo Pettengui, sig, 35.657/13, s/f.

18 Ídem. 